\title{
The two novel MHC class II transactivators RFX5 and CIITA both control expression of HLA-DM genes
}

\author{
Ilse Kern, Viktor Steimle, Claire-Anne Siegrist and Bernard Mach \\ Department of Genetics and Microbiology, University of Geneva Medical School, C.M.U., 9 Av. de Champel, \\ 1211 Geneva 4, Switzerland \\ Keywords: CIITA, gene regulation, HLA-class II, HLA-DM, RFX5
}

\begin{abstract}
MHC-encoded HLA-DMA and -DMB molecules are atypical MHC chains that play an essentlal role in antigen presentation by MHC class II molecules. They resemble both MHC class I and II molecules but are not expressed at the cell surface. From the study of MHC class II regulatory mutants, it was found recently that two novel trensactivators, CIITA and RFX5, are essentlal for the control of MHC class II gene expression. We report here that CIITA and RFX5, although operating at different levels of transcriptlonal control, are also both essentlal regulators of HLA-DMA and -DMB genes. This is true for both the constitutlve and the inducible mode of DM gene expression. Indeed, both CITA and RFX5 CDNA can correct the HLA-DMA and -DMB gene expression defect in the respective regulatory mutants. The Involvement of these two transcription factors accounts for the coordinate expression of MHC class II and HLA-DM, two sets of molecules that perform quite different functions in the overall process of antigen presentation.
\end{abstract}

\section{Introduction}

The $D M A$ and $D M B$ genes were first identified as non-classical $M H C$ genes, mapping to the MHC class II region. From their predicted amino acid sequence, HLA-DM molecules are neither typical MHC class I nor class II molecules, although they show sequence homology to both (1). Their promoters resemble MHC class II promoters (1). The observation that $D M A$ and $D M B$ genes are able to correct certain defects in somatic cell mutants of antigen presentation revealed an interesting role for HLA-DM in the process of assembly of MHC class II-peptide complexes (2,3) and therefore in antigen presentation to CD4 T lymphocytes. DMA and DMB molecules form a stable dimer, which, unlike MHC class II molecules, is not expressed at the cell surface (4). Since MHC class II mediated antigen presentation to CD4 T cells depends on tight control of MHC class II gene expression, the functional role of HLA-DM molecules in antigen presentation obviously raises the issue of the regulation of $D M$ genes, in correlation with that of MHC class II genes.

Our understanding of the mechanisms of MHC class II gene regulation, both constitutive and inducible, and of some of the regulatory factors involved, has benefited from a systematic study of a rare disease, MHC class II deficiency or bare lymphocyte syndrome (BLS) (5-8). In this disease, a total absence of MHC class II expression leads to severe primary immunodeficiency. We have shown that MHC class II deficiency results from distinct regulatory defects, affecting trans-acting regulatory factors (7). Recently two such factors, defective in two distinct forms of BLS (complementation groups $A$ and $C$ ), have been identified by complementation cloning and characterized structurally and functionally $(9,10)$. The MHC class II transactivator CIITA is mutated in patients from group $A$ and transfection of its CDNA into patient cell lines restores normal expression of all class II genes (9). Similarly, RFX5, a DNA binding protein of the RFX family, is mutated in patients from group $\mathrm{C}$; transfection of its CDNA restores MHC class II expression in patient cells (10). Moreover, it has been shown that CIITA is the mediator of inducible expression of MHC class II genes, as in the induction of MHC class II genes by IFN- $\gamma$ (11).

Because of the role of HLA-DM genes in antigen presentation, and despite the fact that HLA-DM molecules are structurally very different from MHC class II molecules, it is of interest to explore whether the expression of HLA-DM genes is also controlled by these two novel MHC class II transactivators. Here we show that CIITA and RFX5 control both constitutive expression of HLA-DM genes in B lymphocytes and their 
expression in cells where MHC class II genes are inducible. Thus the same two regulatory factors, although acting at different levels of transcriptional activation (8), control the expression of both MHC class II and HLA-DMA and -DMB genes.

\section{Methods}

\section{Plasmids construction}

The plasmids used for transfection were EBO-Sfi/CIITA (9), EBO-Sfi (9), EBO-Sfi/RFX5 (10) and SRaNEO/CIITA (V. Steimle and $B$. Mach, unpublished results). The riboprobes used to detect DM mRNA were generated by inserting respectively a BsfXI fragment from DMA (positions 98-477) and a SmaHaell fragment from DMB (positions 233-634) into the EcoRV site of the Bluescript KS vector. The riboprobes for detection of HLA-DRA, CIITA and TATA-binding protein (TBP) mRNA have been previously described $(9,12,13)$.

\section{Cell cultures and transfections}

HeLa cells were grown in Dulbecco's minimum essential medium supplemented with $10 \%$ FCS $10 \mathrm{U} / \mathrm{ml}$ penicillin, $10 \mu \mathrm{g} / \mathrm{ml}$ streptomycin and $2 \mathrm{mM}$ L-glutamine. IFN- $\gamma(500 \mathrm{U} /$ $\mathrm{ml}$ ) was added for the time course experiment. The $B$ lymphoma cell line RAJl and the EBV-transformed B cell lines Mann, BLS-2, SJO, Ro and BLS-1 were grown in RPMI1640 medium also supplemented with $10 \%$ FCS, penicillin, streptomycin and glutamine. Cells were incubated at $37^{\circ} \mathrm{C}$ in $5 \% \mathrm{CO}_{2}$.

BLS-2 transfectant EBO-Sfi/CIITA (9) and SJO transfectants (EBO-Sfi/RFX5 and EBO/Sfi) (10) were generated as described (9). Briefly, cells were transfected by electroporation and hygromycin resistant cells were analyzed by FACS. Stable integrative transfectants were generated with the expression vector SRaNEO/CIITA, with the following modifications to the procedure: HeLa cells were transfected by the $\mathrm{CaPO}_{4}$ method, selected with $\mathrm{G} 418$ and HLA-DR positive cells enriched by sorting with Dynabeads and subsequently cloned by limiting dilution.

\section{RNase protection assays}

Total RNA was extracted from frozen cell pellets by guanidium isothiocyanate lysis and $\mathrm{CsCl}$ step gradient centrifugation (14). To generate riboprobes, the cDNA-containing plasmids were linearized and transcribed with T3 (DMB), T7 (DMA, CIITA) or SP6 RNA polymerase (DRA, TBP) in the presence of [32P]UTP. The specific activity of the DRA and the DM probes was reduced compared with TBP as specified in the figure legends. Hybridization of $5 \mu \mathrm{g}$ of total RNA completed to $50 \mu \mathrm{g}$ with yeast RNA with $100,000-400,000$ c.p.m. of each probe was performed in $80 \%$ formamide, $40 \mathrm{mM}$ PIPES $\mathrm{pH} 6.4,0.4 \mathrm{M} \mathrm{NaCl}$ and $1 \mathrm{mM}$ EDTA, at $50^{\circ} \mathrm{C}$ overnight. The samples were then digested with $23 \mu \mathrm{g}$ RNase A and $3.7 \mathrm{U}$ RNase $\mathrm{T} 1$ for $30 \mathrm{~min}$ at $30^{\circ} \mathrm{C}$. After subsequent treatment with $50 \mu \mathrm{g}$ proteinase $\mathrm{K}$ in the presence of $1 \%$ SDS for $15 \mathrm{~min}$ at $37^{\circ} \mathrm{C}$, the protected RNA fragments were extracted twice with phenol-chloroform, ethanol precipitated and resolved on denaturing $6 \%$ polyacrylamide- $8 \mathrm{M}$ urea gels (15).

\section{Results}

Time course of IFN- $\gamma$ induced expression of the DMA and DMB genes parallels that of the DRA gene

The features of $\mathrm{MHC}$ class II gene induction by IFN- $\gamma$ have been thoroughly studied and are characterized by both a prolonged lag period and dependence on de novo protein synthesis $(12,16,17)$. Although the structure and function of the $D M A$ and $D M B$ genes are quite distinct from those of MHC class $\|$ genes, their promoters are similar in containing the typical class II promoter elements $X$ and $Y$ and it has been shown that HLA-DM expression can be induced by IFN- $\gamma$ (1). It was therefore of interest to further clarify the mechanism of DMA and DMB induction by IFN- $\gamma$, first by a comparison of the time course of their expression in IFN- $\gamma$ treated cells to that of MHC class II genes. RNase protection analysis of the mRNA extracted from HeLa cells incubated between 2 and $72 \mathrm{~h}$ with IFN- $\gamma$ reveals the appearance of $D M A$ and $D M B$ expression after $12 \mathrm{~h}$. The level of expression increases up to $48 \mathrm{~h}$ and remains stable after $72 \mathrm{~h}$ (Fig. 1).

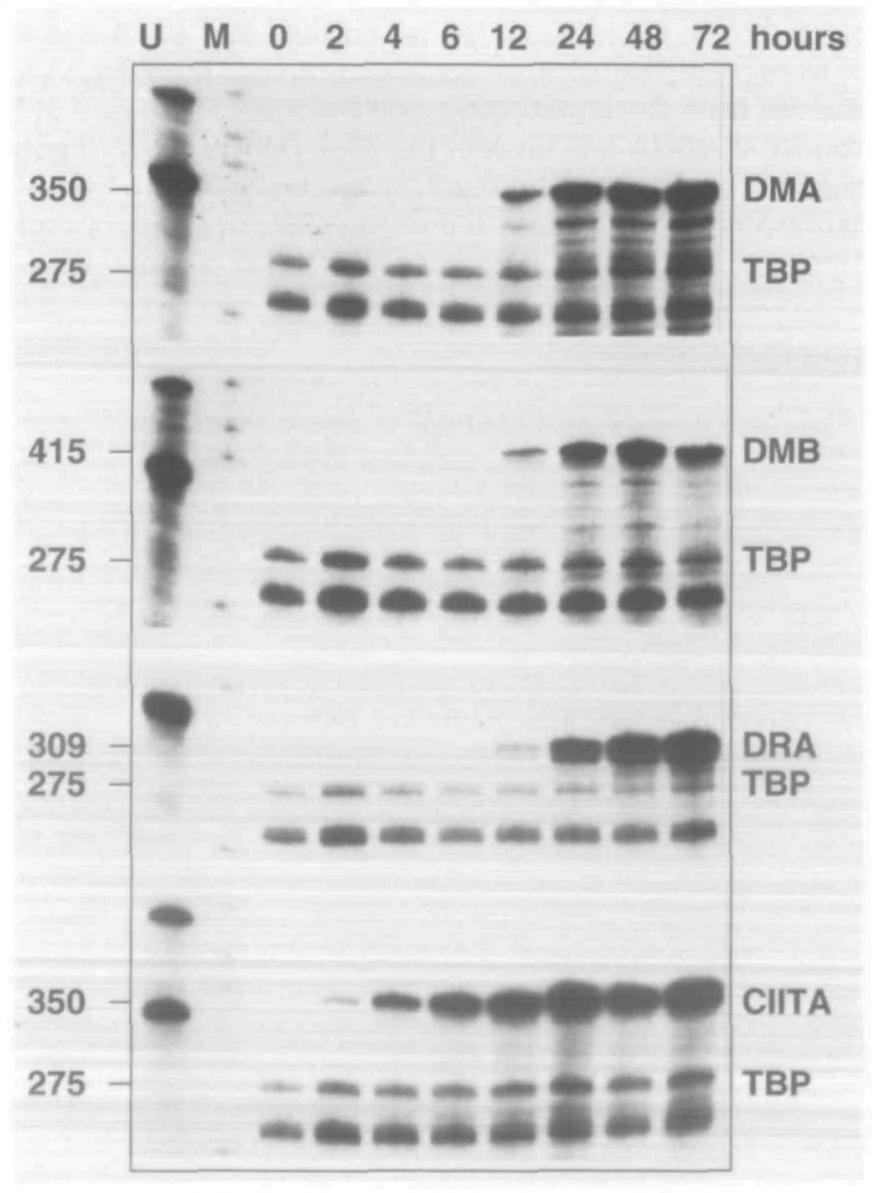

Fig. 1. Time course of HLA-DMA and -DMB mRNA induction in HeLa cells treated with IFN- $\gamma$. The induction of DMA and DMB was compared with that of the IFN- $\gamma$ inducible genes DRA and CIITA. The quantity and quality of the RNA were controlled by hybridization to a TBP specific probe. Specific activity of the ORA probe was reduced fourfold, relative to the other probes. 
The kinetics of $D M A$ and $D M B$ gene induction therefore strictly parallel that of the DRA gene (Fig. 1), with a lag period of 12 $\mathrm{h}$ before first detection of mRNA expression. This contrasts with the time course of IFN- $\gamma$ induced expression of the MHC class II transactivator gene CIITA, which is already detectable $2 \mathrm{~h}$ after addition of IFN- $\gamma$ to the cell culture (Fig. 1).

Constitutive DMA and DMB gene expression is defective in cell lines from patients with $B L S$

HLA-DMA and -DMB genes have been shown to be constitutively expressed in a B lymphoma cell line by Northern blot analysis (1). MHC class II deficiency (BLS) is a genetically heterogeneous disease which can be subdivided into at least three complementation groups, as established by cell fusion experiments $(18,19)$ and by complementation with two of the regulatory genes involved $(9,10)$. It was therefore of interest to analyze the status of HLA-DM gene expression in each of these three complementation groups.

RNase protection analysis of DMA and DMB expression in $B$ cell lines derived from patients with BLS revealed a complete lack of expression in patient BLS-2, representative of complementation group A (Fig. 2, lane 5), Ro and SJO (group C) (Fig. 2, lanes 3 and 4) and BLS-1 (group B) (Fig. 2, lane 2). Lack of expression of DM mRNA in patient SJO had been reported earlier (20). This contrasts with the constitutive

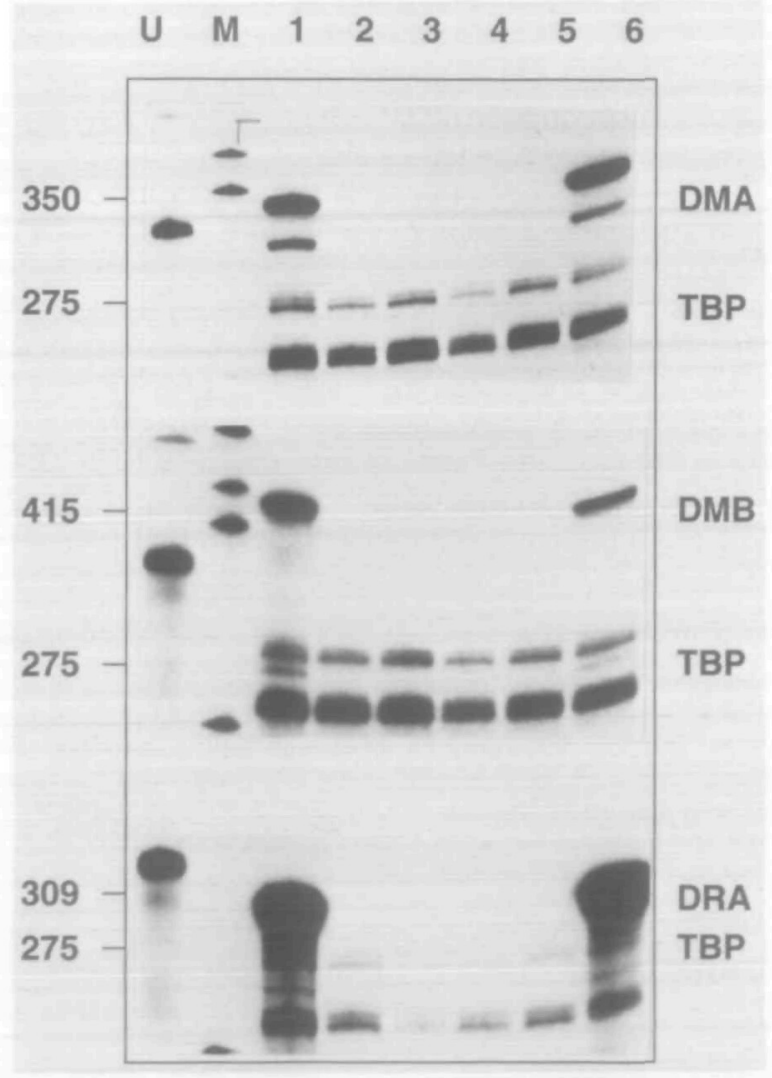

Flg. 2. HLA-DMA and DMB genes are not expressed in cell lines from patients with the BLS. Lane 1: RAJl; lane 2: BLS-1; lane 3: Ro; lane 4: SJO; lane 5: BLS-2; lane 6: Mann. Specific activities of the DM and DRA probes were reduced eight- and 25-fold respectively. expression of the DM genes in the $B$ lymphoma cell line RAJl (Fig. 2, lane 1) and the EBV transformed $B$ cell line Mann (Fig. 2, lane 6). We also confirm the lack of expression of the HLA-DRA gene in all three complementation groups described. We conclude therefore that the transacting defects responsible for each of the three BLS complementation groups also result in the lack of HLA-DM gene expression.

Constitutive DMA and DMB expression can be restored in $B L S$ cell lines by CDNA encoding the regulatory factors CIITA or RFX5

Two molecular and genetic defects responsible for BLS have been recently characterized $(9,10)$. The MHC class II transactivator CIITA is mutated in several cell lines from BLS complementation group A $(9 ; \mathrm{S}$. Bontron, V. Steimle, C. Ucla and $B$. Mach, unpublished results). Transfection of these cell lines with CIITA CDNA allowed restoration of MHC class II

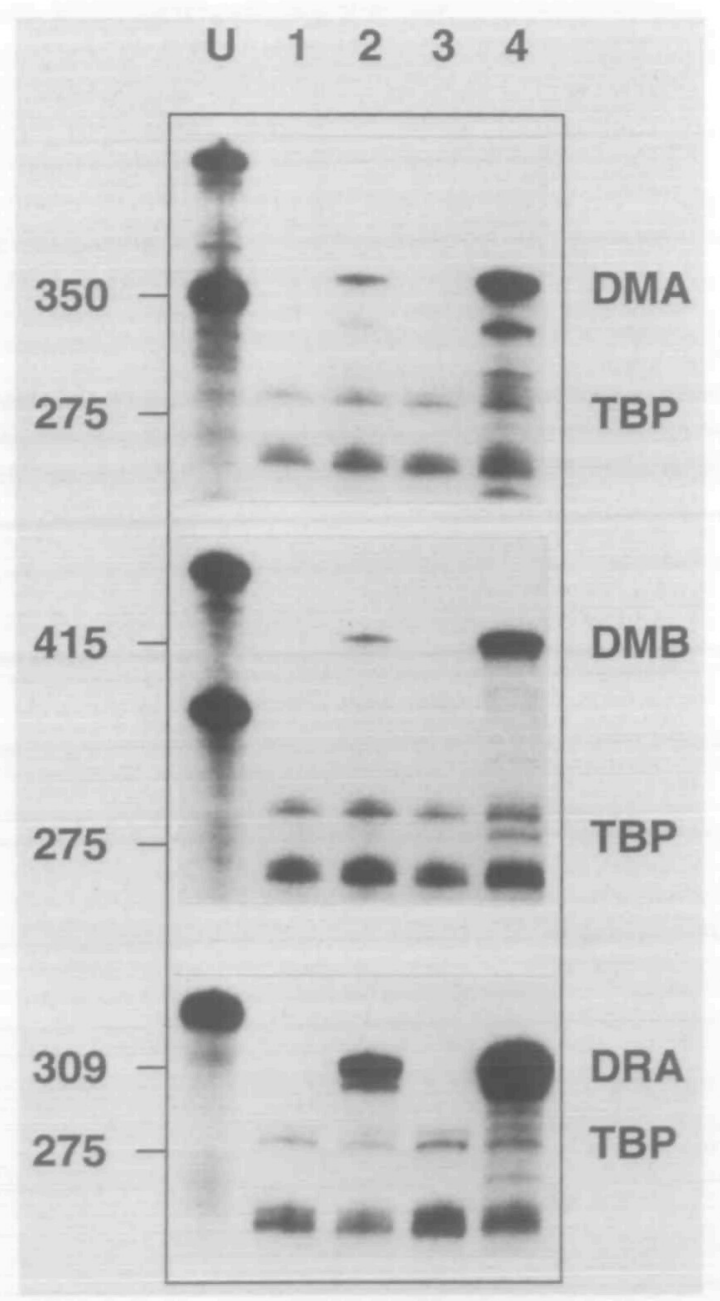

Flg. 3. Restoration of $D M A$ and $D M B$ expression in cell lines from patients with BLS by the relevant regulatory genes. The cell line from patient BLS-2 is corrected by transfection of CIITA CDNA, whereas the cell line from patient SJO is corrected by transfection of RFX5 cDNA Lane 1: BLS-2; lane 2: BLS-2 transfected with CIITA; lane 3: SJO transfected with control plasmid; lane 4: SJO transfected with RFX5. Specific activity of the DRA probe was reduced 25-fold. 
gene expression. Similarly, the transcription factor RFX5 is mutated in three patient cell lines from BLS complementation group C $(10 ;$ W. Reith, J. Villard and B. Mach, unpublished results) and transfection of these cell lines with RFX5 CDNA also restores $\mathrm{MHC}$ class II gene expression. By RNase protection analysis, it was possible to document correction of both DMA and DMB constitutive gene expression in cell lines derived from BLS patients transfected with the relevant regulatory gene (Fig. 3). In the cell line BLS-2, HLA-DM expression is restored through transfection of CIITA CDNA (Fig. 3, lanes 1 and 2). In the cell line SJO, transfection of RFX5 cDNA restores the expression of DMA and DMB (Fig. 3 , lane 4), while transfection of a control plasmid does not (Fig. 3, lane 3). Correction for the expression of the DRA gene is shown as a control.

DMA and DMB gene expression can be induced in HeLa cells by transfection of the MHC class /I transactivator CIITA

The MHC class II transactivator CIITA has been shown to be an essential mediator not only of constitutive but also of inducible MHC class II gene expression (11). Transfection of CIITA CDNA in a variety of MHC class II negative, IFN- $\gamma$ inducible cell lines is sufficient, in the absence of IFN- $\gamma$, to induce expression of MHC class II genes in fibroblastic, monocytic and melanoma cell lines (11).

The effect of CIITA transfection on DMA and DMB gene expression was therefore studied in an IFN- $\gamma$ inducible cell line by quantitative mRNA assays. RNase protection analysis on mRNA from HeLa cells shows the expression of the DMA (Fig. 4, lane 6) and DMB (Fig. 4, lane 4) gene products in stable CIITA transfectants. No DM gene expression can be detected in untransfected cells (Fig. 4, lane 3 and 5). The expression of DRA (Fig. 4, lane 1 and 2) and CIITA MRNA (Fig. 4, lanes 7 and 8 ) is shown as a control of transfection efficiency. Following completion of this study, it was reported elsewhere that CIITA can indeed induce expression of HLA$D M$ in HeLa cells (21). We conclude that both HLA-DMA and $D M B$ genes can be induced by CIITA in these MHC class II negative cells.

\section{Discussion}

The MHC class II locus on the short arm of chromosome 6 contains a cluster of genes encoding structural MHC class II molecules as well as proteins involved in exogenous and

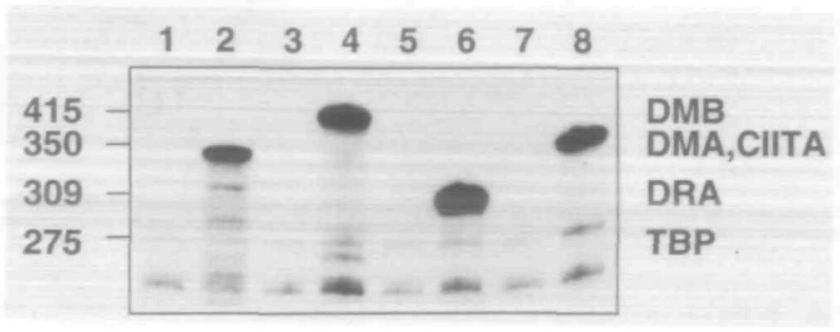

Fig. 4. Transfection of HeLa cells with CIITA CDNA induces DMA and DMB expression. Lanes $1,3,5$ and 7 : untransfected cells. Lanes 2, 4, 6 and 8: CIITA transfected HeLa cells. The specific activities of the DM, DRA and CIITA probes were reduced eight- 40- and twofold respectively, compared with the TBP probe. endogenous antigen processing pathways [reviewed in (22)]. One of the hypotheses put forward to explain the maintenance of this spatial organization is the need for coordinate expression of multiple MHC genes operating at different levels of the complex antigen presentation pathway where all are required to mount an efficient immune response (23).

Our results reveal a coordinate expression of the DMA and $D M B$ genes with the structural MHC class II genes in both constitutive and inducible modes of expression. More importantly, we demonstrate that two essential regulatory factors, recently shown to control MHC class II gene expression, CIITA and RFX5, are both essential for HLA-DM gene expression. First, mutations in either one of these two regulatory genes abolish DM gene expression. More directly, transfection with either CIITA or RFX5 cDNA can restore DM gene expression in regulatory mutants and, in the case of CIITA, induce DM expression in MHC class II negative cells. RFX5 is a transcription factor whose binding to the $X$ box of MHC class II promoters is essential for activity (10), while CIITA does not bind to class II promoters but is required for their transcriptional activity. At these two different levels of transcriptional control, regulation of both $\mathrm{MHC}$ class $\|$ and HLA-DM genes involve, therefore, the same mechanisms.

These mechanisms and transcription factors are very different from those involved in the control of MHC class I gene expression. As expected, MHC class I genes and the MHC encoded proteasome genes, are expressed normally in BLS cells and are thus not controlled by the same transcription factors $(6,20)$.

The role of HLA-DM molecules in antigen presentation and the coordinate regulation of $\mathrm{DM}$ genes with $\mathrm{MHC}$ class II genes can explain the antigen presentation defective phenotype observed with BLS cells transfected only with HLA-DR cDNAs (24). In addition to what is observed in B lymphocytes, and in agreement with the results of Chang and Flavell (21), the data show that expression of CIITA in HeLa cells, without IFN$\gamma$ stimulation, can induce not only MHC class $\|$ genes but also HLA-DMA and -DMB expression. Expression of CIITA, and thus of the different genes controlled by CIITA, in nonprofessional APC can therefore provide these cells with the set of molecules known to be required for an operational MHC class II peptide loading compartment (25).

\section{Acknowledgements}

We are grateful to R. S. Accolla, M. Hadam and J. Lee for generous gifts of BLS cell lines, to J. Trowsdale for DM constructs and to $M$. Zufferey and D. Wohlwend for help with cell culture and FACS analysis. This work was supported by the Swiss National Science Fund and the Louis Jeantet Foundation. I. K. was supported by fellowships from the Sir Jules Thorn Charitable Overseas Trust and the Roche Research Foundation.

\section{Abbreviations}

BLS bare lymphocyte syndrome

TBP TATA-binding protein

\section{References}

1 Kelly, A. P., Monaco, J. J., Cho, S. and Trowsdale, J. 1991. A new human HLA class It-related locus, DM. Nature 353:571. 
2 Fling, S. P., Arp, B. and Pious, D. 1994. HLA-DMA and -DMB genes are both required for MHC class 11/poptide complex formation in antigen-presenting cells. Nature 368:554.

3 Morris, P., Shaman, J., Attaya, M., Amaya, M., Goodman, S., Bergman, C., Monaco, J. J. and Mellins, E. (1994) An essential role for HLA-DM in antigen presentation by class It major histocompatibility molecules. Nature 368:551.

4 Denzin, L. K., Robbins, N. F., Carboy-Newcomb, C. and Cresswell, P. 1994. Assembly and intracellular transport of HLA-DM and correclion of the class 11 antigen-processing defect in T2 cells. Immunity 1:595.

5 Griscelli, C., Lisowska-Grospierre, B. and Mach, B. 1989. Combined immunodeficiency with defective expression in MHC class II genes. Immunodefic. Rev. 1:135.

6 Lisowska-Grospierre, B., Charron, D. J., de Preval, C., Durandy, A., Griscelli, C. and Mach, B. 1985. A defect in the regulation of major histocompatibility complex class $\|$ gene expression in human HLA-DR negative lymphocytes from patients with combined immunodeficiency syndrome. J. Clin. Invest. 76:381.

7 de Preval, C., Lisowska-Grospierre, B., Loche, M., Griscelli, C. and Mach, B. 1985. A trans-acting class II regulatory gene unlinked to the MHC controls expression of HLA class II genes. Nature 318:291.

8 Mach, B., Steimle, V. and Reith, W. 1994. MHC class II-deficient combined immunodeficiency: a disease of gene regulation. Immunol. Rev. 138:207.

9 Steimle, V., Otten, L. A., Zufferey, M. and Mach, B. 1993. Complementation cloning of an MHC class II transactivator mutated in hereditary MHC class II deficiency (or bare lymphocyte syndrome). Cell 75:135.

10 Steimle, V., Durand, B., Barras, E., Zufferey, M., Hadam, M. R., Mach, B. and Reith, W. 1995. A novel DNA-binding regulatory factor is mutated in primary MHC class II deficiency (bare lymphocyte syndrome). Genes Dev. 9:1021.

11 Steimle, V., Siegrist, C.-A., Mottet, A., Lisowska-Grospierre, B. and Mach, B. 1994. Regulation of MHC class II expression by interferon-gamma mediated by the transactivator gene CIITA. Science 265: 106 .

12 Amaldi, I., Reith, W., Berte, C. and Mach, B. 1989. Induction of HLA class II genes by IFN-gamma is transcriptional and requires a trans-acting protein. J. Immunol. 142:999.

13 Kao, C. C., Lieberman, P. M., Schmidt, M. C., Zhou, Q., Pei, R. and Berk, A. J. 1990. Cloning of a transcriptionally active human
Tata binding factor. Science 248:1646.

14 Chirgwin, J. M., Przbyla, A. E., MacDonald, R. J. and Rutter, J. 1979. Isolation of biologically active ribonucleic acid from sources enriched in ribonuclease. Biochemistry 18:5294.

15 Sambrook, J., Fritsch, E. F. and Maniatis, T. 1989. Molecular Cloning: A Laboratory Manual, 2nd edn. Cold Spring Harbor Laboratory Press, Cold Spring Harbor.

16 Blanar, M. A., Boettger, M. C. and Flavell, R. A. 1988. Transcriptional activation of HLA-DR alpha by interferon gamma requires a trans-acting protein. Proc. Natl Acad. Sci. USA 85:4672.

17 Celada, A., Klemsz, M. J. and Maki, A. A. 1989. Interferon-gamma activates multiple pathways to regulate the expression of the genes for major histocompatibility class II I-A beta, tumor necrosis factor and complement component C3 in mouse macrophages. Eur. J. Immunol. 19:1103.

18 Hume, C. R. and Lee, J. S. 1989. Congenital immunodeficiencies associated with absence of HLA class II antigens on lymphocytes result from distinct mutations in trans-acting factors. Hum. Immunol. 26:288.

19 Benichou, B. and Strominger, J. L. 1991. Class II-antigen-negative patient and mutant B-cell lines represent at least three, and probably four, distinct genetic defects defined by complementation analysis. Proc. Natl Acad. Sci. USA 88:4285.

20 Nocera, A., Barocci, S., De Palma, R. and Gorski, J. 1993. Analysis of transcripts of genes located within the HLA-D region in $B$ cells from an HLA-severe combined immunodeficiency individual. Hum. Immunol. 38:231.

21 Chang, C. H. and Flavell, R. A. 1995. Class II transactivator regulates the expression of multiple genes involved in antigen presentation. J. Exp. Med. 181:765.

22 Trowsdale, J. 1993. Genomic structure and function in the MHC Trends Genet. 9:117.

23 Kelly, A. and Trowsdale. J. 1994. Novel genes in the human major histocompatibility complex class-II region. Int. Arch. Allergy Immunol. 103:11.

24 Kovats, S., Drover, S., Marshall, W. H., Freed, D., Whiteley, P. A., Nepom, G. T. and Blum, J. 1994. Coordinate defects in human histocompatibility leukocyte antigen class-II expression and antigen presentation in bare lymphocyte syndrome. J. Exp. Med. 179:2017.

25 Karlsson, L., Péléraux, A., Lindstedt, R., Liljedahl, M. and Peterson, P. A. 1994. Reconstitution of an operational MHC class II compartment in nonantigen-presenting cells. Science 266:1569. 
